\title{
Reliable Routing in Vehicular Ad hoc Networks
}

\author{
Gongjun Yan ${ }^{1} \quad$ Nathalie Mitton ${ }^{2} \quad \mathrm{Xu} \mathrm{Li}^{2}$ \\ ${ }^{1}$ Computer Science Department \\ 2 INRIA/CNRS/University of Lille 1 \\ Old Dominion University, Norfolk, VA 23529, U.S. \\ 59650 Villeneuve d'Ascq, France \\ gyanx001@ odu.edu \\ \{nathalie.mitton,xu.li\}@inria.fr
}

\begin{abstract}
One of the notoriously difficult problems in vehicular ad-hoc networks (VANET) is to ensure that established routing paths do not break before the end of data transmission. This is a difficult problem because the network topology is constantly changing and the wireless communication links are inherently unstable, due to high node mobility. In this paper we classify existing VANET routing protocols into five categories: connectivitybased, mobility-based, infrastructure-based, geographic-locationbased, and probability-model-based, according to their employed routing metrics. For each category, we present the general design ideas and state of the art. Our objective is to attract more attention to the VANET routing problem and encourage more research efforts on developing reliable solutions.
\end{abstract}

Index Terms-Vehicular Ad-hoc Networks, VANET, Routing

\section{INTRODUCTION}

Vehicular Ad-hoc NETwork (VANET) is a form of mobile ad-hoc network (MANET) that provides vehicle-to-vehicle and vhicle-to-roadside wireless communications. It was first introduced by the US Department of Transportation. Indeed, because of its unmistakable societal impact that promises to revolutionize the way we drive, various car manufacturers, government agencies and standardization bodies have spawned national and international consortia devoted exclusively to VANET. Examples include the Car-2-Car Communication Consortium [2], the Vehicle Safety Communications Consortium [3], and Honda's Advanced Safety Vehicle Program [4], among others. The impetus of VANET is that in the notso-distant future vehicles equipped with computing, communication and sensing capabilities will be organized into a ubiquitous and pervasive network that can provide numerous services to travelers, ranging from improved driving safety and comfort (the original goal), to delivering multimedia content on demand, and to other similar value-added services. The initial intention is to provide safety and convenience for passages. Safety-improvement applications are motivated by the need to inform fellow drivers of actual or imminent road conditions, delays, congestion, hazardous driving conditions and other similar concerns. Examples include traffic status reports, collision avoidance, emergency alerts and cooperative driving. The applications such as driver assistance, accident rescue, online payment services, online shopping, and the alike are examples of convenience-improvement applications. Most, if not all, VANET applications involve wireless communications where messages propagate from one vehicle to another.

This paper is an extended version of our recent article [1].
Indeed, the fact of being networked together promotes carto-car communications, even between cars that are tens of miles apart. Imagine, for example, a car that travels down an interstate and whose passengers are interested in viewing a particular movie. The various blocks of this movie happen to be available at various other cars on the interstate, possibly miles away. The task of collecting the blocks of the movie is translated, at the network layer, into finding appropriate routes between the various sources (cars that are willing to share movie blocks) and the receiving car.

It is of paramount importance to develop appropriate VANET routing protocols that dynamically fit the traffic on the fly. Due to short nodal communication range (e.g., mandated by the Federal Communications Commission in the United States), routing paths between cars are usually multi-hop. Cars in various lanes move at different speed, making the underlying network highly dynamic. In such a network, individual communication links are short-lived and the routing paths that rely on a multitude of such links are highly vulnerable to disconnection. It is challenging to realize reliable routing in VANET. Therefore, the motivation of this paper is to introduce the general design ideas of each type of routing method and state of the art of each category of routing protocols. Researchers can combine the features of existing routing protocols into a stronger and more reliable routing protocol that fits their traffic situations.

In literature, a simple routing protocol broadcasts received message to all the neighboring vehicles, called flooding protocol. However, flooding-based routing method occupies the whole network resources and data packets are unnecessarily received by irrelevant nodes. When the number of nodes is large, broadcast storm problem [5] occurs. Thus non-floodingbased light-weighted methods using different routing metrics have been proposed in the literature. With the assistant of fixed infrastructure (i.e., road-side equipments), packets can be propagated among vehicles, even when the traffic is sparse. Infrastructure relays or even buffers packets until next vehicle is available. Use of geographic position was suggested to optimize the routing process. Vehicles knowing the geographic position of neighbors can select a greedy/efficient routing path to transmit packets. Both infrastructure based and geographic position based routing methods need extra device or information. The vehicle mobility is used to predict that if the link between two vehicles will break or not after a certain time interval, in mobility based methods. A probability model based 


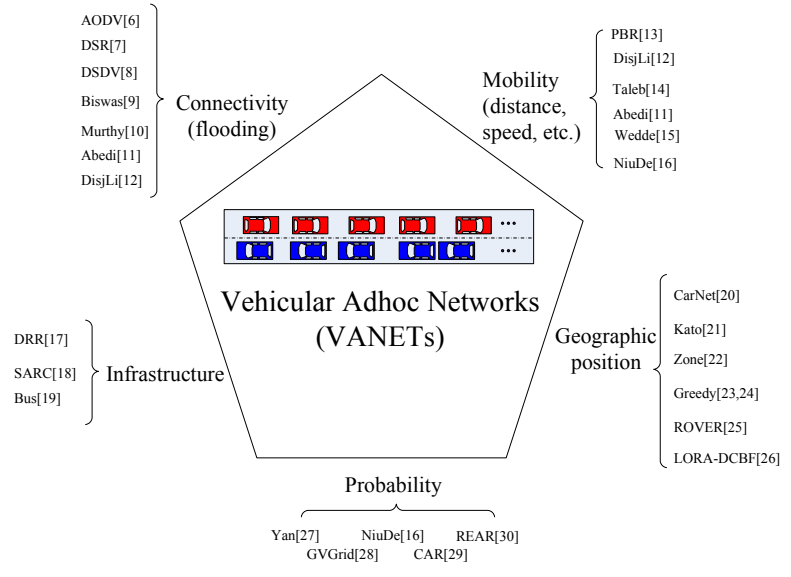

Fig. 1. A taxonomy of VANET routing techniques

routing method avoids using extra device or information. A probability model will be setup to model the wireless routing link which only involves two nodes. The durations of the routing links will be used as a major parameter, stability of a link. The probability based method selectively probes the routing links which compose a routing path, among possible links, to avoid brute-force flooding probing. There are two steps: selectively probing the possible links and selecting a reliable routing path that is composed of multiple routing links.

In the sequel we address the VANET routing problem and survey state of the art of the subject. We present a taxonomy of existing VANET routing methods in Sec. II and describe the general design ideas and representative protocols of each method in Sec. III -VII. We conclude the paper in Sec. VIII.

\section{A TAXONOMY OF VANET Routing}

VANET has several properties that can be exploited for routing. They are connectivity, mobility, infrastructure, geographic location, and probability of its dynamics. According to which property is used, VANET routing techniques can therefore be classified as connectivity-based, mobilitybased, infrastructure-based, geographic-location-based and probability-model-based, as shown in Fig. 1.

Connectivity is formed by enlisting transceivers which can enable the communication network among vehicles. The communication network is a platform to propagate messages. The simplest routing method is based on flooding, where route request messages are broadcasted to every node in the network. There are some well-known flooding-based routing protocols such as AODV [6], DSR [7] and DSDV [8], proposed originally for MANET and extendable to VANET. Some other protocols such as Biswas [9], Murthy [10], Abedi [11] and DisjLi [12] are proposed on the basis of flooding as well.

Mobility is a unique property that does not exist in traditional fixed networks like Ethernet and ATM. It is normally described by relative distance, relative speed, and relative acceleration. Compared with other MANET instances, nodes (i.e., vehicles) in VANET have larger mobility scale (e.g., higher moving speed) and additional mobility constraints (e.g., traffic regulations). They have to follow the directions or moving patterns defined by maps. These mobility features may be used to predict the lifetime/duration of routing paths. PBR [13], DisjLi [12], Taleb [14], Abedi [11], Wedde [15] and NiuDe [16] utilize the mobility parameters to route messages.

In some proposals/implementations of VANET, fixed infrastructure such as Road Side Units (RSU), bridges, buildings, cellular base stations and even routine buses is used. The infrastructure helps to increase the robustness and security of VANET communication. It relays or even buffers packets until next vehicle is available. With the assistance of infrastructure, packets can be propagated among vehicles, even when the traffic is sparse. Protocols such as DRR [17], SARC [18] and Bus [19] adopt fixed infrastructure to propagate messages.

GPS receiver is a handy device in modern vehicles. VANET can use GPS location coordinates to locate other vehicles and to guide vehicles to find destinations (addresses, shops, hotels, etc.). Therefore, geographic location can be used to construct an efficient routing path. There are some geographic-locationbased routing protocols, for example, CarNet [20], Kato [21], Zone [22], Greedy [23], [24], ROVER [25] and LORA-DCBF [26]. They follow the same idea: find the next relay node that is geographically closer to the destination vehicle. They are not concerned about vehicle dynamics induced by high mobility.

Probability theory is often used in dynamical systems to describe the likelihood of certain events, e.g., the probability of link breakage with a certain transmission power or a certain mobility parameter. In a probability-model-based routing protocol, a probability model is first built for the wireless communication link between two nodes. The durations (i.e., stability) of the links in the network will be used as a major routing parameter. The protocol selectively probes, rather than brute-force floods, possible links and selects a reliable multihop routing path. Protocols such as, Yan [27], GVGrid [28], NiuDe [16], CAR [29], and REAR [30] belong to this category.

\section{Connectivity Based Routing}

\section{A. The General Idea}

Connectivity-based routing includes flooding operations as building block. A simple flooding-based routing protocol is as follows. The source node broadcasts a data message containing the identifier of the destination (if any) within its transmission range. A node rebroadcasts the message when it receives the message for the first time. Rebroadcasting proceeds until the destination node is reached or when every node has received a copy of the message. This protocol is easy to implement. It is a good solution for traffic notification applications, event/accident broadcasting applications, etc., which by nature is a flooding process. But, when used for the purpose of unicasting (to a particular destination), it is not efficient.

For applications that need unicast messages, the flooding protocol can be improved by dividing packets into two types: control packets and data packets. The control packets are often RREQ (route request), RERR (route error), and RERP (route reply). RREQ packet spreads out among vehicles, from the 


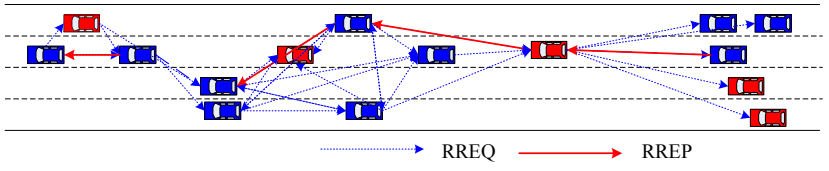

Fig. 2. Connectivity-based routing (the drawing is not proportional)

source. RERP packet returns, from the destination, the selected routing path along all the involved intermediate vehicles. Data packages will then be transmitted along the selected routing path. RERR packet reports the error encountered during path exploration. RREQ and RREP are shown in Fig. 2.

\section{B. State of The Art}

If all nodes can be reached within the transmission range of transceiver, flooding can be seen as an efficient routing scheme. However, in case of multi-hop communication, the performance of network will dramatically drop when the population of nodes increases. Flooding will generate a lot of duplicates of packets and even cause broadcasting storm [5]. In addition, flooding-based routing method scales badly when the network size is beyond a few hundred nodes. But it is a reliable routing in terms of availability, when the network topology is changing and the traffic density is not high.

Since the pure flooding method is costly in delay and bandwidth, enhanced flooding methods are proposed. The basic idea of the enhancement is to find a feasible routing path by broadcasting a control message or probe and then to send packets through the found routing path. For example, Murthy et al. [10] (marked as Murthy) presented a wireless routing protocol by flooding control message over the network that is viewed as a directed graph. AODV ([6]) which is initially proposed for MANET and often extended to VANET, e.g., Abedi [11] and DisjLi [12]. It is a unicast on-demand routing protocol which includes two phases, route discovery and route maintenance. Four types of control packets are used: HELLO, RREQ, RREP, and RERR. Other well-known flooding-based routing protocols include DSR [7] and DSDV [8].

Biswas et al. [9], presented a flooding-based routing method, marked as Biswas, which extends the original flooding method by acknowledging the flooding message. When a node receives a packet, it rebroadcasts the packet. This vehicle watches the packet from following vehicles. If it receives the same message from behind, it infers that at least one vehicle in the back has received the packet and will retransmit the packet. Therefore, the event of receiving the same packet from following vehicles is treated as a acknowledgment of flooding. If the vehicle does not receive the acknowledgment, it will periodically rebroadcast the packet until the acknowledgment is received.

\section{Mobility Based Routing}

\section{A. The General Idea}

Mobility is one of the major differences between VANET and other networks (even other MANET instances). In the wired networks, such as Ethernet and ATM, nodes are fixed in location. In cellular networks, nodes can have fast mobility but the communications among nodes are often through infrastructure, i.e. base station. Conventional MANET is often organized in a small region like airport, and nodes often have slow mobility. Nodes in VANET are often with high mobility. High mobility makes network topology consistently changing and renders communication links and routing path inherently unstable. Traditional routing algorithms are therefore not applicable to VANET. Mobility,

Since mobility is the major reason of the network instability, use of knowledge of the stability of various potential links along the path would naturally help avoid unstable links or links that are about to expire. A routing protocol can be proposed on the basis of link duration (lifetime), vehicle velocity and moving direction. Mobility based routing is reliable when traffic is stable and normal (not jammed or not sparse). It does not work well when the traffic is congested or sparse (mobility predication will not be accurate in this case). Because vehicles have to constantly/periodically send message to each other in order to know the mobility status of neighbors for routing, mobility based routing has extra communication overhead. The reason is that vehicles have to know the status of their neighbors, or neighboring awareness. As the lifetime of a link and the direction of mobility are the two major mobility information used in current research, we introduce them individually in the following two subsections.

1) The lifetime of routing path: If we can predict the lifetime of each communication link, we can maintain a routing path by replacing the links which are about to break. It is natural to compute the lifetime of communication links and predict the lifetime of a routing path. The computation is often on the basis of relationship among time, velocity, acceleration and distance. Suppose two vehicles $i$ and $j$ are the sender and the receiver. Their speeds are $v_{i}$ and $v_{j}$ respectively, and their acceleration are $a_{i}$ and $a_{j}$ respectively. The distance between $i$ and $j$ is $X$ and the speed limit is $v_{m}$. The distance that a vehicle travels in time interval $[0, t]$ is defined as

$$
S(t)=\int_{0}^{t} v(x) d x
$$

where $v(x)$ is the speed at time $x$.

By the above equation, the distances traveled in the time interval $[0, t]$ by vehicle $i$ and $j$ are, respectively, $S_{i}(t)=$ $\int_{0}^{t} v_{i}(x) d x$ and $S_{j}(t)=\int_{0}^{t} v_{j}(x) d x$. Assuming that at connection setup (i.e. time 0 ) the distance between the two vehicles was $d_{0}$, it follows that the distance $d_{t}$ between $i$ and $j$ at time $t$ can be written as

$$
d_{t}=S_{i}(t)-S_{j}(t)+d_{0} .
$$

It is convenient to define the indicator function $I(i, j)$ intended to capture information about which of the two vehicles is ahead when the communication link between them breaks,

$$
I(i, j)= \begin{cases}1 & \text { if } S_{i}(t)-S_{j}(t)+d_{0}>0 \\ -1 & \text { otherwise }\end{cases}
$$

$I(i, j)=1$ indicates that vehicle $i$ is ahead of vehicle $j$; 


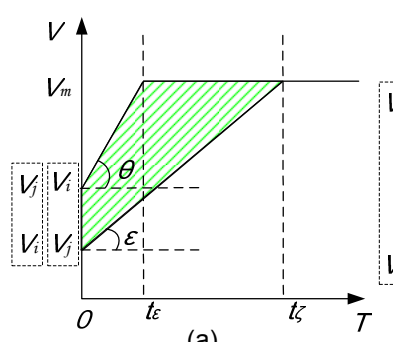

(a)

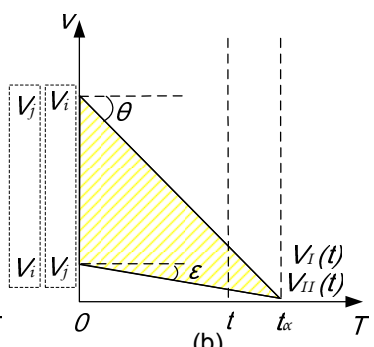

(b)
Fig. 3. The lifetime of communication link

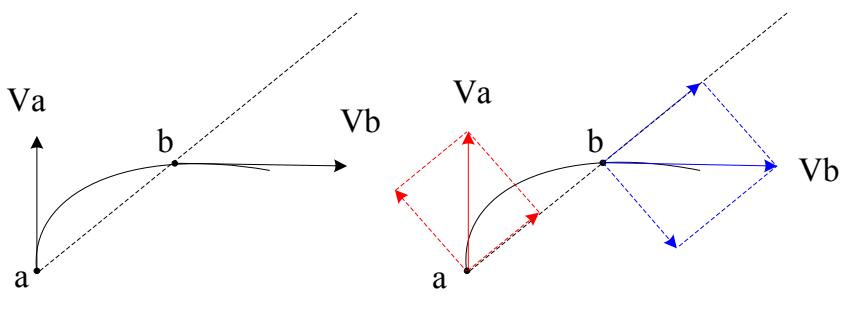

I. before decompose

II. after decompose

Fig. 4. The direction of mobility

otherwise, $j$ is ahead of $i$. Given that a communication link breaks at distant $r$ (the communication range), it follows that when the link breaks the following relation holds:

$$
d_{t}=r \cdot I(i, j) .
$$

The different combinations of $v_{i}, v_{j}, a_{i}$ and $a_{j}$ will change the lifetime of routing links. Two combinations are shown in Fig. 3. Solving Eqn. 4 according to a specific relationship of mobility parameters, we can obtain the link duration/lifetime. The lifetime of the routing path is the minimum lifetime of the all links involved in the routing path.

Once we have link lifetime, we can construct the routing path which is formed by a series of selected routing links. The lifetime of the routing path is the minimum lifetime of the all links involved in the routing path.

2) The direction of mobility: The direction of mobility of two vehicles can be used in routing path selection as well. The basic idea determining the direction of two vehicles is shown in Fig. 4. Suppose two vehicles $a$ and $b$ are moving on speed $v_{a}$ and $v_{b}$. Both $v_{a}$ and $v_{b}$ have directions. We project both speed on the horizontal line which is formed by $a$ and $b$ and the vertical line of the horizontal line. Let $v_{a h}$ and $v_{b h}$ are the horizontal speed projections. Let $v_{a v}$ and $v_{b v}$ are the vertical speed projections. If $v_{a h} \times v_{b h}>0$ and $v_{a r} \times v_{b r}>0$, two vehicles are on the same direction.

\section{B. State of The Art}

Namboodiri et al. [13] present a predictable mobility pattern of vehicles on highway and used it to create a route by predicting the lifetime of route and selecting the best (marked as $P B R$ ). In [12] and [14], a routing method, referred to as Taleb, was presented. The basic idea is to predict a possible

link breakage event prior to its occurrence according to the vehicle's speed. Vehicles are grouped into four different groups based on their velocity vectors (speed with directions). If the directions of speeds of two vehicles are the same, the link between the vehicles will stay longer than the link between two vehicles with different speed directions. The process of routing path search is as follows. Initially, the source node broadcasts a request packet: RREQ. This RREQ will be disseminated among nodes by rebroadcasting. The most suitable (stable) path is chosen when the RREQ reaches the destination. A new route discovery is always initiated prior duration of the routing path, i.e. the shortest link duration.

Abedi et al. [11] presented a enhanced routing protocol (marked as Abedi) based on AODV to adapt the high mobility of vehicles, three mobility parameters: position, direction and speed. This method treats direction as most important parameter to select next hop because the nodes moving with the same directions will be more stable than nodes with opposite directions. Therefore, this method will select routing links composed by nodes with the same mobility directions as the source and/or destination nodes. Moreover, position is the second important parameter that is used for next hop selection. Wedde et al. [15] presented a routing algorithm (marked as Wedde) based on a rating value. The rating value is computed to evaluate the road conditions (actual traffic situation), based on the interdependencies of average vehicle speed, traffic density and the traffic quality (in terms of congestion). A routing link is incorporated into a routing path if the rating value satisfies a certain requirement, i.e. a threshold value. Niu et al. [16] proposed a new link reliability mathematical model (marked as NiuDe) which considers not only the impact of the link duration but also the traffic density. A selected route is not only reliable but also compliant with delay requirements in multimedia application.

\section{INFRASTRUCTURE BASED ROUTING}

\section{A. The General Idea}

Stationary roadside units (RSU) can be combined with onboard units (OBU) equipped on vehicles so as to provide reliable routing and differentiated applications. The basic idea is the following. RSU are used to act as fixed reliable nodes. They are connected by backbone links with high bandwidth, low delay, and low bit error rates. Vehicles communicate with each other by wireless links. Figure 5 gives a picture of this hybrid type of VANET. When the link between two vehicles is broken, RSU will come into play and help to relay packets to the destination vehicle. This routing method is most reliable

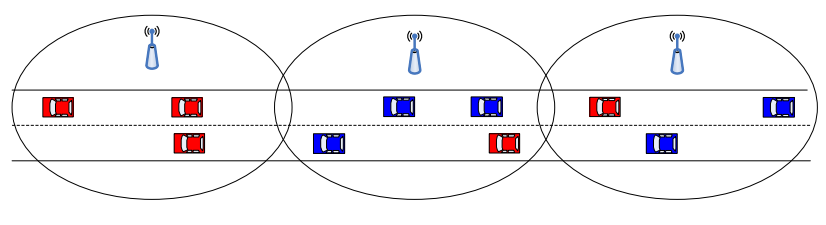

Fig. 5. Road Side Units (RSU) 
and feasible in reality. However, the drawback is that the deployment of infrastructure is costly and limited to urban area. In disasters like hurricane and earthquake where traffic information is most needed, the information may however not be delivered to drivers because the infrastructure is damaged.

\section{B. State of The Art}

$\mathrm{He}$ et al. [17] proposed an algorithm that we refer to as $D R R$. This algorithm introduces two notions, virtual equivalent node (VEN) and differentiated reliable path (DRP) to deal with link failures. If a routing path is broken, RSU (one or multiple) will act as VEN to provide connection to the disconnected clusters of vehicles. After a vehicle successfully connects with an RSU, its position information is synchronized to all related RSU instantly. Kim et al. [18] proposed a routing protocol, called $S A R C$, which can protect privacy in route discovery and data forwarding phase. The protocol adopts a street-based path calculation algorithm for route discovery. The identity, location, and route anonymity are defined and analyzed as well. In [13], accurate prediction of route lifetimes can significantly reduce routing failures.

Kitani et al. [19] proposed an information routing method, marked as Bus, which uses buses as message ferries traveling along regular routes. The motivation of this algorithm is to monitor traffic conditions, like information of traffic jams and road construction sites. These traffic condition information will be transmitted to drivers by FM broadcast and optical beacons on the roadside so that their car navigation systems can display congested areas/roads. Multiple special regions of a road are tagged as "areas". Each vehicle measures time consumption to pass an area. Traffic information statistics can be computed from the information received by cars in intervehicle communication. Buses are ideal media to avoid losing traffic information statistics of areas which buses go along. In the proposed method, buses are assumed to have larger storage than other vehicles and hold traffic information statistics of all areas received from neighboring vehicles. Buses measure time to pass each area by themselves in order to provide traffic information on areas with only a few cars. In order to improve information propagation efficiency in low density areas, buses collect as much traffic information as possible from cars in the communication region, and periodically disseminate the collected information to neighboring cars. Moreover, control packets are transmitted in advance to negotiate the type of information. They can save wireless bandwidth by selecting the specified types of information, instead of sending all information.

\section{Geographic Location BASEd Routing}

\section{A. The General Idea}

Position based routing has received a great deal of attention. They are generally efficient since routing paths can be determined on the fly using location of neighbors and the destination node, without extra route discovery phase. The fundamental idea is the following, as illustrated in Fig. 6. The geographic position of vehicles is used to partition the

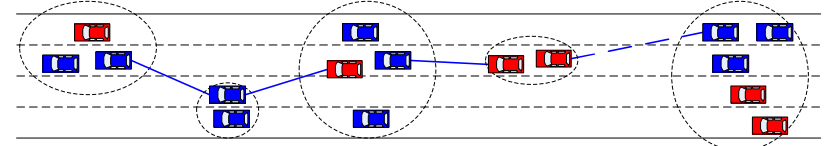

(a)

\begin{tabular}{|c|c|c|c|c|c|c|}
\hline \multirow[t]{2}{*}{ a.1 e6 } & 0. & \multicolumn{2}{|c|}{$\frac{1}{0}$ e 4} & a e3 & e e & $\frac{1}{\mathrm{C}} \mathrm{el}$ \\
\hline & $\mathbf{D}_{\mathrm{f} 7}$ & f5 & f4 & f 3 & f2 & $\mathbf{C}^{\mathrm{fl}}$ \\
\hline
\end{tabular}

(b)

Fig. 6. Geographic-location-based routing. The circles and shaded area represent zones

nodes into sub-sections (groups) along roads. The groups can be dynamically created or predefined. Each group has only one or two vehicles functioning as gateways to send/relay packets. Other members in the group keep silent and drop received packets. This method reduces the number of duplicated packets and therefore, improves the delay and bandwidth utilization. On the other hand, it has message overhead for neighborhood discovery (finding the position of neighbors). In the case of dynamic group creation, it has extra cost for group management. Another drawback is that this method may not find the optimal routing path because relative mobility is not considered.

\section{B. State of The Art}

Morris et al. [20] proposed CarNet based on a grid structure. The grid is defined on the basis of geographic position of nodes. Packets are routed from car to car without flooding the whole network. This algorithm can support many applications: IP connectivity, cooperative highway congestion monitoring, fleet tracking, and discovery of nearby points of interest. In [21], an algorithm, referred to as Kato was proposed for constructing network groups according to lane position and evaluated through simulation. Bronsted et al. [22] presented a zone flooding algorithm and a zone routing algorithm, marked as Zone. A zone is defined as a geographic area, for example, a 500-meter section of a road. If vehicles are in the zone, it allows to broadcast packets; otherwise the received packets will be dropped. The effect of the zone is that packets are only delivered in a section of a road.

Gong et al. [23] and Lochert et al. [24] presented a greedy routing method, referred to as Greedy. As shown in Fig. 6, the basic idea is that vehicles transmit packets aggressively toward the destination (thus "greedy"). Each vehicle knows its own geographic position by GPS or some other methods (e.g., by radio signal strength). Retransmission takes place only at the receiver nodes whose distance to the sender is the longest. The directions of vehicles' movement are taken into consideration during the routing process. It helps to select long-lived links. Mobility direction prediction may also be used to improve the performance.

Kihl et al. [25] described a routing protocol, called ROVER (RObust VEhicular Routing). Zones are defined on the basis of positions of local-aware vehicles. The protocol broadcasts 
control packets, similar to AODV, among zones to find a routing path. Once the routing path is found, data packets are unicasted along the single path. Similarly, [26] proposed a reactive algorithm for MANET, called Location Routing Algorithm with Directional Cluster-Based Flooding (LORADCBF). LORA-DCBF uses zone-like group routing method. To reduce the number of Location Request (LREQ) packets and minimize duplicated retransmissions in the same region, the control packets are selectively transmitted by the selected nodes, called gateways. All the members in the zone can read and process the packet; they do not retransmit. Only gateway nodes retransmit packets between zones, i.e. gateway to gateway communication.

\section{Probability Model Based Routing}

\section{A. The General Idea}

In the presence of frequent topological change, route establishment can be based on, for example, the probability that a wireless link exists between two nodes at a certain time, or the probability that a wireless link stay connected after a certain time intervals. Probability model based routing protocols often make assumptions regarding the model of a certain network parameter or property, and using these assumptions, the distribution of the interested variables can be developed.

One commonly used model is the wireless signal strength attenuation model, which is well-known in wireless communication. The received signal is often assumed to be normally or log-normally distributed. The distribution of the existence of a link can then be computed accordingly. Another popular model is mobility model. Speed and acceleration both are often assumed as normally distributed; distance between two consecutive vehicles can be assumed gamma, normally or lognormally distributed. Under these assumptions, the distribution of link lifetime can be developed.

Probability model based routing is designed to fit certain conditioned traffic. If the condition is satisfied, the protocol is efficient; if the condition is however not satisfied, it may not work or work with lower performance. Another drawback is that a selected routing path may not be the optimal one for a specific sender and receiver pair because the routing metric is the likelihood of an event instead of an accurate measurement.

\section{B. State of The Art}

Jiang et al. [30] presented a routing algorithm, called $R E A R$, based on receipt probability of alarm messages. This algorithm computes the receipt probability of an alarm message based on the real wireless channel in VANET. The selection of next hop is based on the receipt probability. The probability model is grounded on wireless signal strength as well as the loss of signal. The wireless signal loss is composed of two parts: path loss and diffraction loss. The receipt probability is computed by using the relationship between packet loss rate and received signal strength. The receipt probabilities at all neighboring nodes are estimated from the received signal strengths. The path with highest receipt probability is selected for routing.
Niu et al. [16] presented a routing protocol, referred to as $\mathrm{NiuDe}$, which dynamically creates and maintains a robust route to provide QoS for multimedia applications over VANET. The protocol relies on two routing parameters: reliability and delay. The reliability is on the basis of a probability function [31], [32] that predicts the future status of a wireless link. The probability function is defined as the probability that there is an active link between two nodes. The digital map and the GPS device are used to find the route with best reliability. The route is maintained by proactive communication among intermediate nodes. If a link is going to break, the route will be rebuilt before the link breaks.

Yang et. al [29] developed a connectivity aware routing protocol $C A R$. This protocol relies, for routing, on the connectivity probability model of each road segment, which is in turn based on a grid partition on the road segment. The size of each grid cell is the average length of a car, i.e., 5 meters. The probability of the connection between two vehicles is the probability that their distance is within a certain value (transmission range). A route with the highest probability of connectivity will be selected. The packet delivery ratio of CAR is shown quite high, larger than $90 \%$, and routing delay is within acceptable range.

Sun et al. [28] proposed an algorithm referred to as GVGrid to find a reliable routing path meeting given delay requirements. The algorithm assumes that intermediate nodes are equally spaced, and that vehicle speed is normally distributed. Based on these assumptions, it computes the probability of link lifetime as link reliability. By querying possible links or paths, a path with high reliability and sufficiently small link delay will be selected as the routing path.

Yan et al. [27] proposed a ticket based routing algorithm, referred to as Yan, by using the expected link duration. Expected link duration is computed by a probability model. In the same paper, they also proposed a ticket-based probing with stability constrained routing algorithm (TBP-SS), where the routing metric is the mean link duration (defined as stability). Mean link duration is computed also by the probability model. By a divide-and-conquer approach, each optimal routing link is selected, yielding an optimized routing path.

\section{CONCLUSION}

In this paper, we addressed the problem of routing in vehicular ad hoc networks (VANET). On the basis of criteria used for routing, we classified the existing VANET routing methods into five categories. Representative protocols are introduced for each category. Different routing methods have their own features (pros and cons) and applications. Table I gives a brief summary of the routing protocols in VANET. When designing and implementing a VANET routing protocol, one can combine several of these methods for improved performance. For example, probability-model-based routing can be combined with mobility-based routing as the latter can strengthen the the former when the traffic motions change. 
TABLE I

A SUMMARY OF THE ROUTING PROTOCOLS IN VANET

\begin{tabular}{|c|c|c|}
\hline Protocol & Pros & Cons \\
\hline Connectivity & simple & overhead, broadcasting storm \\
\hline Mobility & reliable, accurate & overhead, not working in sparse/congested traffic \\
\hline Infrastructure & reliable, accurate & expensive, not working in rural area \\
\hline Location & simple, direct & overhead, not optimal \\
\hline Probability & efficient & not optimal, only working for a certain traffic \\
\hline
\end{tabular}

\section{REFERENCES}

[1] G. Yan, S. El-Tawab, and D. B. Rawat, Reliable Routing Protocols in VANETs. Mohamed Watfa, Ed. IGI Global, 2009.

[2] Car 2 Car Communication Consortium, http://www.car-to-car.org/.

[3] US Department of Transportation, National Highway Traffic Safety Administration, "Vehicle safety communications consortium," http:// www-nrd.nhtsa.dot.gov/pdf/nrd-12/CAMP3/pages/VSCC.htm.

[4] A. Takahashi and N. Asanuma, "Introduction of Honda ASV-2 (Advanced Safety Vehicle-Phase 2)," in Proceedings of the IEEE Intelligent Vehicles Symposium, Detroit, USA, Oct. 2000, pp. 694-701.

[5] S.-Y. Ni, Y.-C. Tseng, Y.-S. Chen, and J.-P. Sheu, "The broadcast storm problem in a mobile ad hoc network," in Proceedings of the 5th annual ACM/IEEE international conference on Mobile computing and networking, Seattle, Washington, United States, 1999, pp. 151-162.

[6] C. E. Perkins, E. M. Belding-Royer, and S. R. Das, "Ad hoc on-demand distance vector (aodv) routing," RFC Experimental 3561, July 2003. [Online]. Available: http://rfc.net/rfc3561.txt

[7] D. B. Johnson, D. A. Maltz, and Y. C. Hu, "The dynamic source routing protocol for mobile ad hoc networks (dsr)," Published Online, IETF MANET Working Group, Tech. Rep., February 2007.

[8] C. E. Perkins and P. Bhagwat, "Highly dynamic destination-sequenced distance-vector routing (dsdv) for mobile computers," SIGCOMM Comput. Commun. Rev., vol. 24, no. 4, pp. 234-244, 1994.

[9] S. Biswas, R. Tatchikou, and F. Dion, "Vehicle-to-vehicle wireless communication protocols for enhancing highway traffic safety," Communications Magazine, IEEE, vol. 44, no. 1, pp. 74-82, Jan. 2006.

[10] S. Murthy and J. J. Garcia-Luna-Aceves, "An efficient routing protocol for wireless networks," Mob. Netw. Appl., vol. 1, no. 2, pp. 183-197, 1996.

[11] O. Abedi, M. Fathy, and J. Taghiloo, "Enhancing aodv routing protocol using mobility parameters in vanet," in Proceedings of the 2008 IEEE/ACS International Conference on Computer Systems and Applications (AICCSA08). Washington, DC, USA: IEEE Computer Society, 2008, pp. 229-235.

[12] X. Li and L. Cuthbert, "On-demand node-disjoint multipath routing in wireless ad hoc network," in Proceedings of the 29th Annual IEEE International Conference on Local Computer Networks (LCNO4). Washington, DC, USA: IEEE Computer Society, 2004, pp. 419-420.

[13] V. Namboodiri and L. Gao, "Prediction-based routing for vehicular ad hoc networks," Vehicular Technology, IEEE Transactions on, vol. 56, no. 4, pp. 2332-2345, July 2007 .

[14] T. Taleb, E. Sakhaee, A. Jamalipour, K. Hashimoto, N. Kato, and Y. Nemoto, "A stable routing protocol to support its services in vanet networks," Vehicular Technology, IEEE Transactions on, vol. 56, no. 6, pp. 3337-3347, Nov. 2007.

[15] H. F. Wedde, S. Lehnhoff, and B. van Bonn, "Highly dynamic and scalable vanet routing for avoiding traffic congestions," in Proceedings of the fourth ACM international workshop on Vehicular ad hoc networks (VANET07). New York, NY, USA: ACM, 2007, pp. 81-82.

[16] Z. Niu, W. Yao, Q. Ni, and Y. Song, "Dereq: a qos routing algorithm for multimedia communications in vehicular ad hoc networks," in Proceedings of the 2007 international conference on Wireless communications and mobile computing (IWCMC07). New York, NY, USA: ACM, 2007 , pp. 393-398.

[17] R. He, H. Rutagemwa, and X. Shen, "Differentiated reliable routing in hybrid vehicular ad-hoc networks," May 2008, pp. 2353-2358.

[18] H. Kim, J. Paik, B. Lee, and D. Lee, "Sarc: A street-based anonymous vehicular ad hoc routing protocol for city environment," in Proceedings of the 2008 IEEE/IFIP International Conference on Embedded and Ubiquitous Computing (EUC08). Washington, DC, USA: IEEE Computer Society, 2008, pp. 324-329.

[19] T. Kitani, T. Shinkawa, N. Shibata, K. Yasumoto, M. Ito, and T. Higashino, "Efficient vanet-based traffic information sharing using buses on regular routes," May 2008, pp. 3031-3036.

[20] R. Morris, J. Jannotti, F. Kaashoek, J. Li, and D. Decouto, "Carnet: A scalable ad hoc wireless network system," in Proceedings of the 9th ACM SIGOPS European workshop: Beyond the PC: New Challenges for the Operating System. ACM Press, 2000, pp. 61-65.

[21] T. Kato, K. Kadowaki, T. Koita, and K. Sato, "Routing and address assignment using lane/position information in a vehicular ad hoc network," in Proceedings of the 2008 IEEE Asia-Pacific Services Computing Conference (APSCC08). Washington, DC, USA: IEEE Computer Society, 2008, pp. 1600-1605.

[22] J. Bronsted and L. Kristensen, "Specification and performance evaluation of two zone dissemination protocols for vehicular ad-hoc networks," April 2006, pp. 12 pp.-.

[23] J. Gong, C.-Z. Xu, and J. Holle, "Predictive directional greedy routing in vehicular ad hoc networks," in Proceedings of the 27th International Conference on Distributed Computing Systems Workshops (ICDCSW07). Washington, DC, USA: IEEE Computer Society, 2007, p. 2.

[24] C. Lochert, H. Hartenstein, J. Tian, H. Fussler, D. Hermann, and M. Mauve, "A routing strategy for vehicular ad hoc networks in city environments," June 2003, pp. 156-161.

[25] M. Kihl, M. Sichitiu, T. Ekeroth, and M. Rozenberg, Reliable Geographical Multicast Routing in Vehicular Ad-Hoc Networks. Springer Berlin / Heidelberg, 2007.

[26] S. Momeni and M. Fathy, "Vanet's communication," Aug. 2008, pp. 608-612.

[27] G. Yan, S. Olariu, and S. Salleh, "A probabilistic routing protocol in vanet," in Proceedings of the 7th International Conference on Advances in Mobile Computing and Multimedia (MoMM2009), Kuala Lumpur, Malaysia, December 14-16 2009.

[28] W. Sun, H. Yamaguchi, and K. Yukimasa, "Gvgrid: A qos routing protocol for vehicular ad hoc networks," in Proceedings of the Fourteenth IEEE International Workshop on Quality of Service (IWQoS 2006), Yale University, New Haven, CT, USA, June 19-21 2006, pp. 130-139.

[29] Q. Yang, A. Lim, and P. Agrawal, "Connectivity aware routing in vehicular networks," 31 2008-April 3 2008, pp. 2218-2223.

[30] H. Jiang, H. Guo, and L. Chen, "Reliable and efficient alarm message routing in vanet," in Proceedings of the 2008 The 28th International Conference on Distributed Computing Systems Workshops (ICDCSW08). Washington, DC, USA: IEEE Computer Society, 2008, pp. 186-191.

[31] I. Rubin and Y.-C. Liu, "Link stability models for qos ad hoc routing algorithms," vol. 5, Oct. 2003, pp. 3084-3088 Vol.5.

[32] S. Jiang, D. He, and J. Rao, "A prediction-based link availability estimation for mobile ad hoc networks," vol. 3, 2001, pp. 1745-1752 vol.3. 